Bundesgesundheitsbl 2015 $\cdot 58: 560-568$

DOI 10.1007/s00103-015-2157-y

Online publiziert: 14. April 2015

(c) Springer-Verlag Berlin Heidelberg 2015
Anna Kuehne - Lena Fiebig · Klaus Jansen - Carmen Koschollek · Claudia Santos-Hövener

Abteilung für Infektionsepidemiologie, Robert Koch-Institut, Berlin, Deutschland

\title{
Migrationshintergrund in der infektionsepidemiologischen Surveillance in Deutschland
}

\author{
Analysen am Beispiel Tuberkulose, \\ HIV und Syphilis
}

\section{Hintergrund}

Etwa $20 \%$ der Bevölkerung Deutschlands haben einen Migrationshintergrund [1]. Der Ausdruck „Menschen mit Migrationshintergrund“ beschreibt Menschen, deren Leben durch ihre eigene Migrationserfahrung oder die der Herkunftsfamilie geprägt ist [2]. Migrationshintergrund kann entsprechend definiert werden als das Tragen einer ausländischen Staatsangehörigkeit, das Vorhandensein eigener Migrationserfahrung oder die Einwanderung von mindestens einem Elternteil nach Deutschland [1].

\section{Operationalisierung \\ Migrationshintergrund}

Migrationserfahrungen und -situationen können sich in vielfältiger Weise unterscheiden. Zur Auswertung von epidemiologischen Daten ist deshalb die alleinige Erhebung von deutscher oder nichtdeutscher Herkunft in aller Regel unzureichend [2]. Vielmehr wird für die Erfassung von verschiedenen Facetten der Migrationserfahrung ein Indikatorensatz empfohlen, der Geburtsland, Geburtsland der Eltern, Aufenthaltsdauer, Muttersprache und Staatsangehörigkeit bzw. Aufenthaltsstatus umfasst [2]. Diese Mindestindikatoren finden derzeit unter anderem in repräsentativen epidemiologischen Bevölkerungssurveys in Deutschland Anwendung [3].
Sowohl in Deutschland als auch in anderen Mitgliedsstaaten der Europäischen Union (EU) bzw. des Europäischen Wirtschaftsraums (EWR) wird der Migrationshintergrund bei ausgewählten Infektionskrankheiten erhoben und ausgewertet. Auf europäischer Ebene wird die Erhebung von „Geburtsland“ als Indikator für Migrationshintergrund in Surveillance-Daten empfohlen $[4,5]$.

\section{Migration und \\ Infektionskrankheiten}

Ein Migrationshintergrund kann in mehrfacher Hinsicht einen Risikofaktor für bestimmte Infektionskrankheiten darstellen. In vielen Ländern mit niedrigerem Lebensstandard sind Infektionskrankheiten die Hauptursache für Morbidität und Mortalität [6-8]. Zusätzlich zu einem möglicherweise erhöhten Infektionsrisiko im Herkunftsland können der Migrationsprozess selbst sowie die Situation im Aufnahmeland, z. B. durch eine Unterbringung in Gemeinschaftsunterkünften, mit einem erhöhten Risiko für Expositionen und Infektionsszenarien im Zusammenhang stehen $[5,9]$. Dennoch sind Migranten ${ }^{1}$ nicht grundsätzlich häufiger von Infektionskrankheiten betroffen [10]. Die Kenntnis über eine Migrationserfahrung

1 Zur Erhöhung der Lesbarkeit wird im Folgenden ausschließlich die männliche Form verwendet, sie bezieht sich jedoch auf Angehörige aller Geschlechtsidentitäten. oder einen Migrationshintergrund bei Vorliegen einer Infektionskrankheit kann jedoch dabei helfen, Infektionsrisiken gezielt für bestimmte Bevölkerungsgruppen einzuschätzen und darauf aufbauend diagnostische und präventive Maßnahmen $\mathrm{zu}$ initiieren.

\section{Zielstellung}

Ziel des vorliegenden Beitrags ist eine systematische Darstellung von Indikatoren zur Operationalisierung des Migrationshintergrundes in der infektionsepidemiologischen Surveillance in Deutschland sowie die Einschätzung bestehender Einschränkungen.

\section{Material und Methoden}

Diese Übersichtsarbeit bezieht sich auf die gemäß Infektionsschutzgesetz (IfSG) in Deutschland meldepflichtigen Infektionskrankheiten, für die Informationen zum Migrationshintergrund verfügbar sind. Für alle meldepflichtigen Krankheiten bzw. Nachweise von Krankheitserregern, bei denen Daten zum Migrationshintergrund erhoben werden, werden die jeweils genutzten Indikatoren und deren gesetzliche Grundlage dargestellt.

Am Beispiel von Tuberkulose (TB), HIV und Syphilis erfolgt eine deskriptive Auswertung der verfügbaren migrations-

Anna Kuehne und Lena Fiebig sind beide Erstautorinnen. 
Tab. 1 Verfügbare Informationen zum Migrationshintergrund in den Meldedaten nach Infek tionsschutzgesetz (IfSG)

\begin{tabular}{|c|c|c|c|c|c|}
\hline $\begin{array}{l}\text { Informationen zum Migrationshinter- } \\
\text { grund in den Meldedaten nach IfSG }\end{array}$ & $\begin{array}{l}\text { Tuber- } \\
\text { kulose }\end{array}$ & $\begin{array}{l}\text { HIV- } \\
\text { Infektion }\end{array}$ & Syphilis & Malaria & $\begin{array}{l}\text { Hepati- } \\
\text { tis A }\end{array}$ \\
\hline "Staatsangehörigkeit ${ }^{u a}$ & $x$ & & & & \\
\hline "Geburtsland" & $\mathrm{x}$ & & & & \\
\hline $\begin{array}{l}\text { "Geburtsland der Eltern bei Kindern } \\
\text { unter } 15 \text { Jahren" }\end{array}$ & $x^{b}$ & & & & \\
\hline „Herkunft Hochprävalenzgebiet" & & $x$ & & & \\
\hline $\begin{array}{l}\text { "Herkunft Hochprävalenzgebiet" als } \\
\text { zusätzliche „Information zum Risiko der } \\
\text { vermuteten Infektionsquelle" }\end{array}$ & & $x$ & & & \\
\hline „Herkunftsland des Patienten“ & & $x$ & $x$ & $x$ & \\
\hline Angabe des Aufenthaltsstatus & & & & $x$ & \\
\hline $\begin{array}{l}\text { Angabe einer Region unter "Migrations- } \\
\text { hintergrund" }\end{array}$ & & & & & $\mathrm{X}$ \\
\hline \multicolumn{6}{|c|}{$\begin{array}{l}\text { aln Anführungszeichen markierte Formulierungen geben wörtlich die Formulierungen in den Meldebögen ge- } \\
\text { mäß IfSG bzw. der Meldesoftware SurvNet3@RKI wider } \\
\text { bDie Übermittlung wurde ab Mitte } 2011 \text { ermöglicht }\end{array}$} \\
\hline
\end{tabular}

Übersicht 1 Definitionen der in der Surveillance verwendeten Indikatoren zur Erfassung des Migrationsstatus

Geburtsland: Land, in dem der Patient geboren wurde. Anzugeben ist der Staat, in dessen Grenzen der Geburtsort zum Zeitpunkt der Ermittlung liegt (d. h. nach heute gültiger Grenzziehung) [11].

Staatsangehörigkeit: Die Staatsangehörigkeit ist definiert als Staatsangehörigkeit zum Zeitpunkt der Einleitung der Behandlung laut Ausweis (Mehrfachnennung möglich) [11].

Herkunftsland: Das Herkunftsland ist das Land, in dem sich die betreffende Person Zeit ihres Lebens überwiegend aufgehalten hat. Dies muss nicht mit dem Geburtsland oder der Staatsangehörigkeit übereinstimmen [12].

Hochprävalenzland: Land, in dem HIV endemisch ist ( $>1 \%$ der heterosexuellen Allgemeinbevölkerung) und überwiegend heterosexuell übertragen wird [13].

spezifischen Angaben aus der RoutineSurveillance des Robert Koch-Institutes (RKI) aus dem Zeitraum 2002 bis 2013 in Deutschland.

Angaben zum Migrationshintergrund wurden entweder dichotom (Deutschland vs. alle anderen Länder), aggregiert zu Regionen der Weltgesundheitsorganisation (WHO) oder disaggregiert auf der Ebene einzelner Staaten ausgewertet. Zur Berechnung von Meldeinzidenzen wur- den die Bevölkerungsstatistiken der Statistischen Landesämter vom 31. Dezember der entsprechenden Jahre verwendet.

Die Datenerhebung und -übermittlung für TB durch lokale Gesundheitsämter erfolgte mittels der jeweils verwendeten Meldesoftware, die Übermittlung auf Ebene der Landesstellen und am RKI ausschließlich mittels der Software SurvNet3@RKI. Für HIV und Syphilis werden Daten direkt per Meldebogen an das RKI gemeldet. Die Datensätze liegen dem RKI auf gesetzlicher Grundlage des IfSG nichtnamentlich vor.

\section{Ergebnisse}

\section{Infektionskrankheiten und genutzte migrationsspezi- fische Indikatoren}

Im Rahmen der Surveillance von Infektionskrankheiten gemäß IfSG werden in Deutschland nur bei TB, HIV, Syphilis, Malaria und Hepatitis A Informationen über den Migrationshintergrund erhoben.

Nicht für alle oben genannten Infektionskrankheiten werden identische Indikatoren zur Bestimmung des Migrationshintergrundes genutzt (• Tab. 1). Die zur Erhebung der Indikatoren verwendeten Definitionen sind in (• Übersicht 1) dargestellt.

Informationen über Geburtsland und Staatsangehörigkeit bei Fällen von TB werden gemäß $₫ 9$ Absatz 1 Nr. 10 sowie $₫ 11$ Absatz 1 Nr. 8 IfSG erhoben und übermittelt. Das Geburtsland der Eltern bei Meldungen von TB (bei gemeldeten Erkrankungen bei Kindern < 15 Jahre) und der Migrationshintergrund bei Meldungen von Hepatitis A kann gemäß $\$ 11$ Absatz 1 Nr. 6 IfSG übermittelt werden. Für Meldungen von Malaria, HIV und Syphilis werden zur Beschreibung des Infektionsrisikos Indikatoren für einen $\mathrm{Mi}$ grationshintergrund gemäß $₫ 10$ Absatz 1 Nr. 9 IfSG verwendet.

\section{Auswertung der Meldedaten}

\section{Tuberkulose}

Im Zeitraum von 2002 bis 2013 wurden in Deutschland 64.048 TB-Fälle gemeldet und an das RKI übermittelt. Bei 61.532 Patienten waren Angaben zum Geburtsland vorhanden. Von diesen waren $54 \%$ in Deutschland geboren, $46 \%$ in einem von insgesamt 166 anderen Staaten. Die häufigsten ausländischen Geburtsländer waren die Türkei (6\%), die Russische Föderation (4\%), Kasachstan (3\%), Indien (2\%), Serbien (2\%), Polen (2\%) und Rumänien (2\%). In WHO-Regionen gruppiert, was für 61.259 Fälle möglich war, war Europa (81\%) am häufigsten genannt, gefolgt vom Östlichen Mittelmeerraum (6\%), Afrika (5\%), Südostasien (4\%), Westpazifik (3\%) und Amerika (0,6\%). Über die Beobachtungszeit verringerten sich die Fallzahlen insgesamt. Der Anteil der Patienten, die in Deutschland geboren waren, sank von $58 \%$ auf $43 \%$. Dies war vor allem durch einen stärkeren Rückgang der Fallzahlen unter in Deutschland Geborenen bedingt. Unter im Ausland Geborenen $(N=28.164)$ sank der Anteil an in der Türkei oder in den Neuen Unabhängigen Staaten der ehemaligen Sowjetunion (NUS) geborenen Patienten von $15 \%$ auf $9 \%$ bzw. von $24 \%$ auf $17 \%$. Steigende Fallzahlen und Anteile wurden nur für die WHO-Regionen Östlicher Mittelmeerraum (von $12 \%$ auf 19\%) und Südostasien (von $7 \%$ auf $11 \%$ ) verzeichnet. In Deutschland geborene TB-Patienten hatten mit 58 Jahren den höchsten Altersmedian.

Der Anteil multiresistenter Fälle unter in NUS geborenen TB-Patienten war mindestens 6,5-mal so hoch wie in allen 
Bundesgesundheitsbl 2015 · 58:560-568 DOI 10.1007/s00103-015-2157-y

(c) Springer-Verlag Berlin Heidelberg 2015

\section{A. Kuehne · L. Fiebig · K. Jansen · C. Koschollek · C. Santos-Hövener}

\section{Migrationshintergrund in der infektionsepidemiologischen Surveillance in Deutschland. Analysen am Beispiel Tuberkulose, HIV und Syphilis}

\section{Zusammenfassung}

Hintergrund. Migration beeinflusst die Epidemiologie von Infektionskrankheiten. Sich daraus ergebende Risikogruppen und Präventionsbedarfe zu identifizieren, ist Grundlage für eine adäquate Gestaltung von Public-Health-Maßnahmen. Es stellt sich die Frage, inwieweit sich migrationsspezifische Informationen hierfür direkt aus der infektionsepidemiologischen Surveillance ableiten lassen.

Ziel der Arbeit. Ziel ist eine systematische Darstellung von Indikatoren zur Operationalisierung des Migrationshintergrundes in der infektionsepidemiologischen Surveillance in Deutschland sowie die Einschätzung bestehender Einschränkungen.

Methodik. Für meldepflichtige Krankheiten bzw. Erregernachweise werden die jeweils er- hobenen Indikatoren für Migration und deren Grundlage im Infektionsschutzgesetz dargestellt. Für Tuberkulose (TB), HIV und Syphilis werden Meldedaten für 2002-2013 deskriptiv analysiert.

Ergebnisse. Bei fünf Infektionskrankheiten wurden - unterschiedlich operationalisiert - Informationen zum Migrationshintergrund erhoben. Bei TB (Geburtsland) und HIV (Herkunftsland) war eine nicht-deutsche Herkunft deutlich häufiger als bei Syphilis (Herkunftsland) mit 46, 30 bzw. $13 \%$ der Fälle mit entsprechenden Angaben. Bei allen drei betrachteten Infektionskrankheiten ergaben sich Hinweise auf migrationsspezifische Risikoprofile.

Diskussion. Einheitliche Indikatoren für Migration in der infektionsepidemiologischen
Surveillance würden die internationale und erregerübergreifende Vergleichbarkeit der Daten ermöglichen. Die Surveillance erlaubt aktuell teilweise migrationssensible Analysen, jedoch bedarf es zusätzlicher Studien, um die komplexen Zusammenhänge von $\mathrm{Mi}$ gration und Infektionskrankheiten richtig interpretieren und Public-Health-Maßnahmen bedarfsgerecht gestalten zu können.

\section{Schlüsselwörter}

Infektionskrankheiten · Migration · Migrant . Meldewesen · Indikatoren

\section{Migration and infectious disease surveillance in Germany. Analyses of Tuberculosis, HIV and Syphilis surveillance data}

\begin{abstract}
Background. Migration is an important factor impacting on infectious disease epidemiology. The timely identification of groups at risk and prevention needs resulting from migration is indispensable to adequately design and implement public health measures. It remains to be assessed to which extent surveillance data for notifiable diseases can directly generate meaningful migration-specific information.

Objectives. The objectives of this study are to review indicators of migration background utilized in the German infectious disease surveillance, as well as to assess their limitations. Methods. We describe the indicators of migration used for mandatorily notifiable dis-
\end{abstract}

eases and pathogens and their legal basis in the Protection against Infection Act and conduct a descriptive analysis of surveillance data for tuberculosis (TB), HIV and syphilis from 2002-2013.

Results. Migration status is collected only for five infectious diseases and operationalization varies. For TB (country of birth) and HIV (country of origin) a foreign origin was more frequent than for syphilis (country of origin); namely 46,30 and $13 \%$ of cases with available information, respectively. In all three examples, there are indications of risk profiles that are specific for particular groups of migrants.
Discussion. A standardization of indicators of migration in infectious disease surveillance is important to enhance data comparability between diseases and pathogens as well as across countries. Routine surveillance already partly allows migration sensitive analyses, yet further research is needed to guide interpretation of the complex relationship between migration and infectious diseases and plan public health measures adequately.

\section{Keywords}

Infectious diseases · Migration · Migrant .

Surveillance $\cdot$ Indicators anderen Geburtsländern bzw. -regionen (- Tab. 2).

Neben dem Geburtsland wird von TBPatienten auch die Staatsangehörigkeit erfasst. Im Zeitraum von 2002 bis 2013 lag für 63.442 Patienten eine Angabe zur Staatsangehörigkeit vor. Diese war in $63 \%$ der Fälle die deutsche Staatsangehörigkeit und in $37 \%$ der Fälle eine andere Staatsangehörigkeit. Die durchschnittliche Meldeinzidenz für TB betrug 6,5 Erkrankungen pro 100.000 Einwohner. In der ausländischen Bevölkerung war diese über 5-mal höher als in der deutschen Bevölkerung (23,4 vs. 4,4 Erkrankungen pro 100.000 Einwohner).

Insgesamt stimmten die Angaben zu Staatsangehörigkeit und Geburtsland (dichotom betrachtet) in $88 \%$ (53.438/60.827) der Fälle überein. In 11\% der Fälle waren Erkrankte im Ausland geborene Deutsche, jeder zweite von ihnen war in NUS geboren. In 1\% der Fälle wurden Deutschland als Geburtsland und eine ausländische Staatsangehörigkeit angegeben.
Bei 135 von 333 Kindern unter 15 Jahren mit Angaben zum eigenen Geburtsland (41\%) waren Informationen zum Geburtsland von zumindest einem Elternteil vorhanden; $52 \%$ aller Kinder mit einem nicht-deutschen Vater oder einer nicht-deutschen Mutter waren selber in Deutschland geboren (Daten für 2012 und 2013).

\section{HIV}

Im Zeitraum von 2002 bis 2013 wurden in Deutschland 31.212 HIV-Erstdiag- 
Tab. 2 In Deutschland gemäß IfSG gemeldete TB-Fälle nach ausgewählten Charakteristika stratifiziert nach WHO-Regionen und häufigsten Geburtsländern, 2002-2013 (gepoolt)

\begin{tabular}{|c|c|c|c|c|c|c|c|c|}
\hline \multirow{2}{*}{$\begin{array}{l}\text { Geburtsland gruppiert } \\
\text { nach WHO-Regionen }\end{array}$} & \multicolumn{2}{|c|}{ Alter (in Jahren) } & \multicolumn{2}{|l|}{ Geschlecht } & \multicolumn{4}{|l|}{ Resistenzen ${ }^{\mathbf{b}}$} \\
\hline & Median & $\begin{array}{l}\text { Interquar- } \\
\text { til-Abstand }\end{array}$ & $\begin{array}{l}\text { Geschlecht, } \\
\text { Angaben (N) }\end{array}$ & Frauen (\%) & $\begin{array}{l}\text { Jegliche Resistenz, } \\
\text { Angaben }(N)\end{array}$ & $\begin{array}{l}\text { Jegliche Re- } \\
\text { sistenz (\%) }\end{array}$ & $\begin{array}{l}\text { Multiresistenz, } \\
\text { Angaben }(N)\end{array}$ & $\begin{array}{l}\text { Multiresis- } \\
\text { tenz (\%) }\end{array}$ \\
\hline Europa $(N=49.707)$ & 53 & {$[38-70]$} & 49.660 & 38,1 & 32.608 & 12,1 & 32.605 & 2,3 \\
\hline Deutschland $(N=33.368)$ & 58 & [42-73] & 33.350 & 37,5 & 21.598 & 8,3 & 21.603 & 0,7 \\
\hline$N U S^{a}(N=5.809)$ & 45 & [31-63] & 5801 & 36,4 & 4001 & 35,7 & 3987 & 13,7 \\
\hline Türkei $(N=3873)$ & 45 & [32-61] & 3868 & 41,9 & 2411 & 12,4 & 2414 & 0,6 \\
\hline $\begin{array}{l}\text { Sonstige Länder } \\
(N=6657)\end{array}$ & 46 & [32-62] & 6641 & 40,8 & 4598 & 9,2 & 4601 & 1,2 \\
\hline Afrika $(N=3111)$ & 30 & [24-38] & 3106 & 42,9 & 2265 & 14,2 & 2265 & 2,1 \\
\hline Amerika $(N=388)$ & 33 & [27-42] & 387 & 62,0 & 297 & 15,8 & 297 & 2,0 \\
\hline $\begin{array}{l}\text { Östlicher Mittelmeer- } \\
\text { raum }(N=3556)\end{array}$ & 34 & [25-49] & 3546 & 39,7 & 2359 & 14,9 & 2361 & 1,4 \\
\hline Südostasien $(N=2675)$ & 32 & [26-40] & 2664 & 45,3 & 1264 & 22,2 & 1977 & 1,6 \\
\hline Westpazifik ( $N=1822)$ & 34 & [26-44] & 1815 & 57,3 & 174 & 12,1 & 1264 & 2,1 \\
\hline
\end{tabular}

aNUS Neue unabhängige Staaten der ehemaligen Sowjetunion

${ }^{\mathrm{b}} M D R$ Multiresistenz; d. h. Resistenz gegenüber mindestens Isoniazid und Rifampicin; jegliche Resistenz = Resistenz gegenüber mindestens einem der Standard-Antituberkulotika (Isoniazid, Rifampicin, Ethambutol, Pyrazinamid und Streptomycin)

nosen an das RKI gemeldet. Unter allen Fällen war bei $59 \%$ Deutschland und bei $26 \%$ ein anderes Land als Herkunftsland angeben. Bei $15 \%$ der Fälle lagen keine Angaben zu Herkunftsland oder -region vor. Im Folgenden beziehen wir uns auf die Grundgesamtheit der Personen, bei denen ein Herkunftsland bzw. eine Herkunftsregion angeben wurde $(n=26.457)$. Das am häufigsten genannte Herkunftsland war Deutschland (70\%), gefolgt von der Russischen Föderation (2\%), Thailand (2\%) und Kamerun (2\%). Häufigste Herkunftsregionen unter den HIVNeudiagnosen waren die WHO-Regionen Europa ( $81 \%)$ und Afrika (11\%). Unter den Personen mit Herkunft aus der WHO-Region Afrika stammten $99 \%$ aus einem Land der Subsahara-Region.

Im Rahmen der HIV-Meldungen wird der wahrscheinliche Infektionsweg erfragt. Diese Information war bei 21.735 der Fälle mit bekanntem Herkunftsland verfügbar. Männer, die Sex mit Männern haben (MSM), stellten mit $68 \%$ die größte Gruppe der HIV-Neudiagnosen. Bei $93 \%$ der MSM war die WHO-Region Europa bzw. bei $85 \%$ aller MSM das Herkunftsland Deutschland angegeben. Bei intravenösen Drogengebrauchern war die Herkunftsregion bei $95 \%$ die WHO-Region Europa, $61 \%$ stammten aus Deutschland. Heterosexuelle Übertragung wurde bei $26 \%$ der Fälle als wahrscheinlicher
Infektionsweg angegeben. Die häufigsten Herkunftsländer bzw. -regionen waren die WHO-Region Afrika (48\%) und die WHO-Region Europa (37\%) einschließlich Deutschland (30\% aller Angaben). Bei heterosexueller Übertragung galt für alle Herkunftsregionen, dass mehr Frauen unter den Neudiagnostizierten waren als Männer.

Die Anteile von Personen, die bereits in ihrer jeweiligen Herkunftsregion ihre HIV-Infektion erwarben, variierten, ein jeweils substanzieller Anteil dieser Infektionen wurde aber in der WHO-Region Europa akquiriert (• Tab. 3). Insgesamt $38 \%$ aller Infektionen bei Personen nichtdeutscher Herkunft wurden in Deutschland erworben. Die absolute Zahl von HIV-Neudiagnosen bei Personen deutscher und europäischer Herkunft nahm über die Zeit leicht zu (• Abb. 1).

\section{Syphilis}

Zwischen 2002 und 2013 wurden 40.484 Syphilis-Diagnosen an das RKI gemeldet. Für $66 \%$ dieser Fälle wurde eine Angabe zum Herkunftsland gemacht. Diese Fälle bilden die Grundlage für die folgenden Analysen $(n=26.557)$. Bei $87 \%$ der Fälle wurde Deutschland als Herkunftsland angegeben, in $13 \%$ ein anderes Land, am häufigsten die Russische Föderation (1\%), die Türkei (1\%), Polen und Italien (jeweils $0,8 \%$ ) sowie Bulgarien und $\mathrm{Ru}$ - mänien (jeweils 0,7\%). Die WHO-Region Europa (inklusive Deutschland) entsprach mit $96 \%$ der am häufigsten angegebenen Herkunftsregion. Alle anderen WHO-Regionen spielten eine untergeordnete Rolle: Auf die WHO-Region Amerika (2\%) folgten die WHO-Regionen Östlicher Mittelmeerraum (0,9\%), Südostasien $(0,6 \%)$, Westpazifik $(0,5 \%)$ und Afrika (0,3\%).

Mit einem medianen Alter von 39 Jahren waren Personen deutscher Herkunft älter als Personen nicht-deutscher Herkunft (34 Jahre); der Frauenanteil betrug $6 \%$ bzw. $19 \%$. Auch die Verteilung der gemeldeten wahrscheinlichen Infektionswege unterschied sich zwischen Personen mit Herkunft aus Deutschland oder aus dem Ausland: Infektionen von Deutschen wurden zu $83 \%$ wahrscheinlich durch Sex zwischen Männern erworben, weitere $17 \%$ auf heterosexuellem Weg. Demgegenüber machte Sex zwischen Männern bei Nicht-Deutschen $67 \%$ aller Fälle aus, heterosexuelle Übertragungen $33 \%$. Bei $3 \%$ der Fälle mit deutscher Herkunftsangabe wurde Kontakt zu Prostituierten als wahrscheinlicher Infektionsweg angegeben, in weiteren $0,9 \%$ Ausübung von Prostitution. Bei Fällen mit anderer Herkunft betrug der Anteil mit Angabe eines Kontakts zu Prostituierten 5\%, mit Angabe der Ausübung von Prostitution als wahrscheinlichem Infektionsweg $7 \%$. 
Tab. 3 Anzahl der in Deutschland gemäß IfSG gemeldeten HIV-Erstdiagnostizierten: Herkunftsregion ${ }^{\mathrm{a}}$ stratifiziert nach Infektionsregionen, 2002-2013 (gepoolt)

\begin{tabular}{|c|c|c|c|c|c|c|c|}
\hline \multirow{2}{*}{$\begin{array}{l}\text { Herkunftsland gruppiert } \\
\text { nach WHO-Regionen }\end{array}$} & \multicolumn{7}{|c|}{ Infektionsland gruppiert nach WHO-Regionen } \\
\hline & Afrika & Europa & $\begin{array}{l}\text { Östlicher } \\
\text { Mittelmeerraum }\end{array}$ & Amerika & Südostasien & Westpazifik & unb \\
\hline & $N=2315$ & $N=19.990$ & $N=155$ & $N=372$ & $N=670$ & $N=154$ & $N=2422$ \\
\hline & $(8,9 \%)$ & $(76,7 \%)$ & $(0,6 \%)$ & $(1,4 \%)$ & $(2,6 \%)$ & $(0,6 \%)$ & $(9,3 \%)$ \\
\hline & $n(\%)$ & $n(\%)$ & $n(\%)$ & $n(\%)$ & $n(\%)$ & $n(\%)$ & $n(\%)$ \\
\hline \multirow[t]{2}{*}{ Afrika } & 2090 & 428 & 3 & 2 & 1 & 1 & 418 \\
\hline & $(71,0 \%)$ & $(14,5 \%)$ & $(0,1 \%)$ & $(0,1 \%)$ & $(0,0 \%)$ & $(0,0 \%)$ & $(14,2 \%)$ \\
\hline \multirow[t]{2}{*}{ Europa } & 221 & 18.762 & 27 & 150 & 319 & 46 & 1.778 \\
\hline & $(1,0 \%)$ & $(88,1 \%)$ & $(0,1 \%)$ & $(0,7 \%)$ & $(1,5 \%)$ & $(0,2 \%)$ & $(8,3 \%)$ \\
\hline \multirow[t]{2}{*}{ Östlicher Mittelmeerraum } & 1 & 179 & 124 & 1 & 2 & 0 & 42 \\
\hline & $(0,3 \%)$ & $(51,3 \%)$ & $(35,5 \%)$ & $(0,3 \%)$ & $(0,6 \%)$ & $(0,0 \%)$ & $(12,0 \%)$ \\
\hline \multirow[t]{2}{*}{ Amerika } & 3 & 369 & 0 & 218 & 0 & 0 & 82 \\
\hline & $(0,4 \%)$ & $(54,9 \%)$ & $(0,0 \%)$ & $(32,4 \%)$ & $(0,0 \%)$ & $(0,0 \%)$ & $(12,2 \%)$ \\
\hline \multirow[t]{2}{*}{ Südostasien } & 0 & 139 & 0 & 0 & 347 & 3 & 75 \\
\hline & $(0,0 \%)$ & $(24,6 \%)$ & $(0,0 \%)$ & $(0,0 \%)$ & $(61,5 \%)$ & $(0,5 \%)$ & $(13,3)$ \\
\hline \multirow[t]{2}{*}{ Westpazifik } & 0 & 113 & 1 & 1 & 1 & 104 & 27 \\
\hline & $(0,0 \%)$ & $(45,7 \%)$ & $(0,4 \%)$ & $(0,4 \%)$ & $(0,4 \%)$ & $(42,1 \%)$ & $(10,9 \%)$ \\
\hline
\end{tabular}

Häufigste außerdeutsche Herkunftsländer von Personen in der Prostitution waren Rumänien (20\%), Bulgarien (16\%), Polen (11\%) sowie die Russische Föderation und Thailand (jeweils 5\%).

Angaben zum Land, in dem die Syphilis-Infektion erworben wurde, lagen für $72 \%$ der Fälle aus den Jahren 2002 bis 2013 vor. Bei $93 \%$ dieser Fälle wurde die Infektion in Deutschland erworben, in $7 \%$ in einem andern Land, am häufigsten in Spanien $(0,9 \%)$, in der Russischen Föderation $(0,7 \%)$, Thailand $(0,5 \%)$ und in der Tschechischen Republik (0,4\%). Der wahrscheinliche Infektionsweg MSM wurde deutlich häufiger bei Fällen mit Infektionsland Deutschland angegeben $(82 \%)$ als bei im Ausland erworbenen Infektionen (48\%). Der Anteil von Fällen mit heterosexuellem Übertragungsweg betrug für in Deutschland erworbene Infektionen $17 \%$, für außerhalb Deutschlands akquirierte Infektionen $51 \%$.

\section{Diskussion}

Der vorliegende Beitrag gibt erstmals einen umfassenden Überblick über verwendete Indikatoren zur Operationalisierung des Migrationshintergrundes in der infektionsepidemiologischen Surveillance in Deutschland am Beispiel von TB, HIV und Syphilis. Hierzu wurden die in den deutschen Surveillance-Daten verfügbaren Informationen zum Migrationshintergrund über den Zeitraum 2002 bis 2013 systematisch ausgewertet und verglichen.

\section{Indikatoren zur Operationalisierung des Migrationshintergrundes}

Aktuell werden in Deutschland nur bei ausgewählten Infektionskrankheiten bzw. Erregern Angaben zum Migrationshintergrund erhoben. Die erhobenen Indikatoren unterscheiden sich dabei sowohl zwischen verschiedenen Infektionskrankheiten als auch für die jeweiligen Infektionskrankheiten im europäischen Vergleich.

Auf europäischer Ebene wird das Geburtsland als verlässlichster Indikator zur Beschreibung eines Migrationshintergrunds empfohlen [5, 14]. Es wird in über $80 \%$ der EU/EWR-Mitgliedsstaaten im Rahmen der infektionsepidemiologischen Routine-Surveillance von TB und HIV erhoben, in Deutschland nur für TB. Zusätzlich wird es in einigen EU/EWR-
Mitgliedsstaaten für Fälle von Syphilis, Hepatitis B und C sowie Gonorrhö erfasst $[4,5]$.

Der in der HIV- und Syphilis-Surveillance verwendete Indikator Herkunftsland wird europaweit unterschiedlich definiert. Für diesen Indikator kann nur schlecht sichergestellt werden, dass er von den Meldenden einheitlich verstanden wird (z. B. als Geburtsland oder Land des längsten Aufenthalts über die Lebensspanne) sowie international vergleichbar ist. Daher wird er weder vom Europäischen Zentrum für die Prävention und Kontrolle von Krankheiten (ECDC) empfohlen noch in der Erhebung von Bevölkerungsdaten verwendet $[1,14,15]$. Die direkte Berechnung von Inzidenzen ist nicht möglich. Bei Krankheiten mit peripartaler Übertragung und langen Inkubationszeiten und/oder Krankheitsverläufen ist die Information des Geburtslandes zudem relevant für die Einschätzung des Infektionsrisikos.

Im Gegensatz zum Geburtsland ist die Staatsangehörigkeit ein veränderlicher Indikator: Personen können ihre Staatangehörigkeit wechseln oder mehrere Staatsangehörigkeiten tragen. Allerdings ermöglicht die Erhebung der Staatsange- 


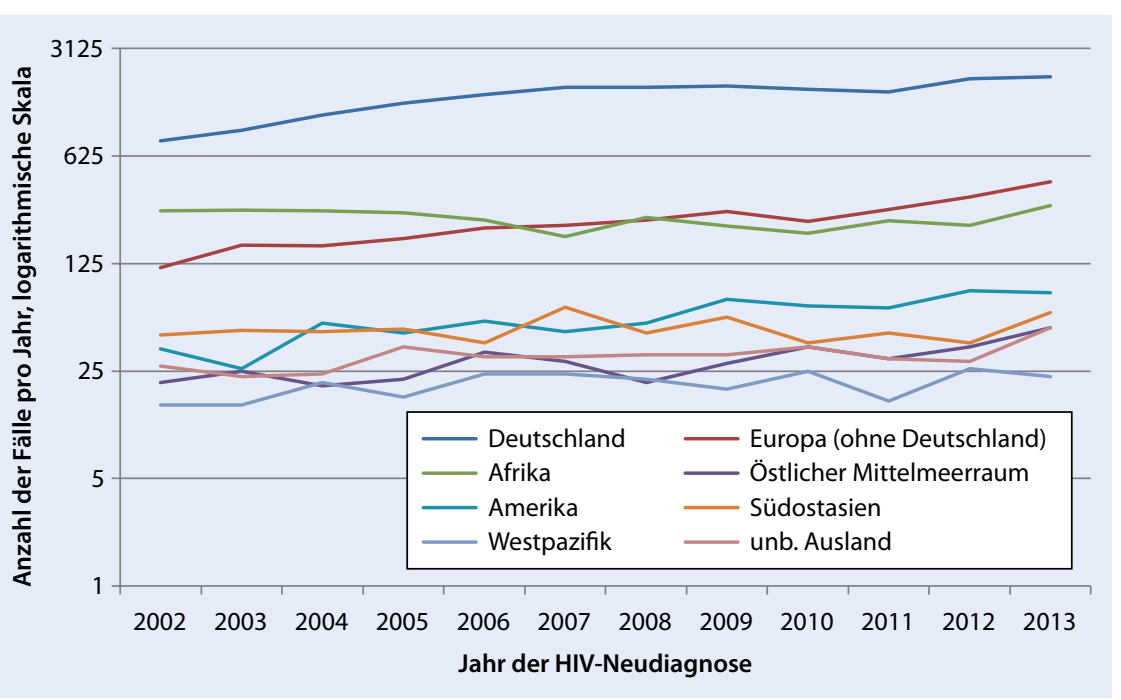

Abb. $1 \Delta$ Anzahl der in Deutschland gemäß IfSG gemeldeten HIV-Erstdiagnostizierten nach Herkunftsland, gruppiert nach WHO-Regionen (Deutschland separat und ohne fehlenden Angaben), 2002-2013

hörigkeit die Berechnung von Inzidenzen und Prävalenzen, da Bevölkerungsdaten, die z. B. im Rahmen des Mikrozensus erhoben werden, die Staatsangehörigkeit erfassen $[1,15]$.

Die Gegenüberstellung des Geburtslandes und der Staatsangehörigkeit bei TB zeigt jedoch in Übereinstimmung mit der Literatur, dass die alleinige Angabe der Staatsangehörigkeit die Bedeutung von Migration für das TB-Geschehen in Deutschland untererfassen würde $[11,16]$. Auch die gleichzeitige Angabe von Geburtsland und Staatsangehörigkeit stellt eine Untererfassung eines jeglichen Migrationshintergrundes dar. Eine Studie in Berlin zeigte, dass in Deutschland geborene Personen mit mindestens einem im Ausland geborenen Elternteil ein im Vergleich zu Personen ohne Migrationshintergrund erhöhtes TB-Risiko hatten [17]. Dies unterstreicht die Bedeutung der initiierten Erfassung des Geburtslandes der Eltern bei Kindern unter 15 Jahren im Rahmen der TB-Surveillance.

Nachteile der Verwendung uneinheitlicher Indikatoren für verschiedene Infektionskrankheiten werden deutlich, wenn Koinfektionen bzw. Komorbiditäten untersucht werden sollen, zum Beispiel von HIV und TB. So konnten in vergleichenden Auswertungen Aussagen zu HIV und HIV/TB nur auf das Herkunftsland bezogen, die Epidemiologie der TB jedoch nur in Bezug auf das Geburtsland beschrieben werden $[18,19]$.

Weitere empfohlene Indikatoren wie Aufenthaltsdauer, Muttersprache, Ethnie und Angaben über regelmäßige Bematland („visiting friends and relatives“ VFR) [2, 20] werden in der infektionsepidemiologischen Surveillance derzeit nicht erhoben, obwohl Daten hierzu helfen könnten, vulnerable Gruppen genauer zu identifizieren und Präventionsangebote kultursensibel und mehrsprachig zu gestalten.

Für eine Aufnahme von Indikatoren des Migrationsstatus in die RoutineSurveillance müssen ihre epidemiologische Relevanz und Operationalisierbarkeit belegt sein sowie der Aufwand bei der Datenerfassung durch die Meldenden berücksichtigt werden. Grundsätzlich ist eine Angabe von migrationsspezifischen Daten nur bei Krankheiten und Erregernachweisen möglich, die durch den behandelnden Arzt zu melden sind ( $\$ 6$ IfSG) oder bei denen der einsendende Arzt den Meldepflichtigen in der Regel unterstützt ( $\$ 10$ IfSG).

Die betrachteten Beispiele, insbesondere die hohe Vollständigkeit der Angaben zu den Indikatoren für einen Migrationshintergrund bei TB und HIV, sprechen für die grundsätzliche Umsetzbarkeit migrationssensibler infektionsepidemiologischer Surveillance. Neben der suche von Familie und Freunden im Hei-
Auswahl geeigneter und vergleichbarer Indikatoren bleibt die adäquate Interpretation eine Herausforderung.

Migrationshintergrund in der Routine-Surveillance - Daten für Taten?

\section{Tuberkulose}

Die Surveillance belegt, dass TB in Deutschland bei Personen mit und ohne Migrationserfahrung vorkommt. Im Meldejahr 2013 hatte mehr als die Hälfte der TB-Patienten ein nicht-deutsches Geburtsland. Der Anteil an einheimischen TB-Patienten ist jedoch verglichen mit Norwegen und Schweden mehr als doppelt so hoch [5]. Das Spektrum an nichtdeutschen Geburtsländern unter TB-Patienten ist groß, was in der Migrationsstruktur in Deutschland insgesamt begründet ist [1]. Die EU/EWR-weite Beobachtung, dass ein Drittel der migrantischen TB-Patienten eine Herkunft aus Asien hat, spiegelt sich in Deutschland nicht wider. In Deutschland beobachtete Tendenzen, wie eine angestiegene Zahl an TB-Patienten mit Geburtsland in der WHO-Region Östlicher Mittelmeerraum, werden wiederum nicht notwendigerweise in EU/EWR-Statistiken deutlich [5]. Veränderungen in der Zusammensetzung der Herkunftsregionen der TB-Patienten stehen mit Wanderungsbewegungen in Zusammenhang. Allerdings kann eine TB sowohl direkt nach der Einreise, z. B. bei Aufnahme in eine Gemeinschaftseinrichtung, diagnostiziert und gemeldet werden, als auch bei Personen mit Migrationserfahrung auftreten, die seit langer Zeit in Deutschland leben. In einer Studie in Berlin vergingen bei TB-Patienten mit eigener Migrationserfahrung im Median 8 Jahre (Spannbreite 0 bis 51 Jahre) zwischen der Einreise und der TB-Diagnose [17]. In der bundesweiten Surveillance ist diese Angabe nicht verfügbar.

Im Ausland geborene TB-Patienten waren jünger als in Deutschland geborene. Gemäß berechneter altersspezifischer Inzidenzen nach Staatsangehörigkeit in der TB-Berichterstattung ist dies nicht allein auf unterschiedliche Altersstrukturen zurückzuführen; es besteht tatsächlich ein höheres Erkrankungsrisiko unter jüngeren Erwachsenen mit ausländischer Staatsangehörigkeit [11]. Die Anteile resis- 
tenter und multiresistenter TB unterscheiden sich nach Geburtsregion bzw. -land. Hierbei ging jedoch ein nicht-deutsches Geburtsland nicht grundsätzlich mit einem hohen Anteil an multiresistenter TB einher, sondern spezifisch die Geburtsregion NUS.

Zeitnahe Auswertungen der TB-Surveillance-Daten stratifiziert nach spezifischen Geburtsregionen bzw. -ländern sind daher nicht nur für eine migrationssensible Ausrichtung der TB-Fürsorge, sondern auch im Hinblick auf Diagnoseund Therapieentscheidungen informativ.

\section{HIV}

Der Aspekt der Migration ist wesentlich für die Betrachtung der HIV-Epidemie in Deutschland. Bei mindestens einem Viertel der HIV-Neudiagnosen wurde in den letzten Jahren ein anderes Herkunftsland als Deutschland angegeben. Wichtigste Herkunftsregionen waren die WHO-Regionen Europa und Afrika.

Obwohl die Population der Personen aus der Subsahara-Region in Deutschland lediglich bei< $0,1 \%$ der Gesamtbevölkerung liegt, hatte diese Gruppe unter den heterosexuell übertragenen HIV-Infektionen den größten Anteil [21]. Auch wurde jährlich bei mindestens $15 \%$ der Personen aus der Subsahara-Region angegeben, dass sie sich in Europa - meist in Deutschland - mit HIV infiziert haben. Möglicherweise war dieser Anteil tatsächlich höher als erfasst, da der Indikator Infektionsland auf der Annahme des meldenden Arztes beruht und den tatsächlichen Anteil der Personen, die sich innerhalb des Ziellandes mit HIV infiziert haben, vermutlich unterschätzt [22]. Somit besteht für diese Gruppe ein Bedarf für Primärprävention. Vor diesem Hintergrund wird am RKI zurzeit die MiSSAStudie durchgeführt (• Tab. 4), die die Ermittlung der spezifischen Präventionsbedarfe dieser Population zum Ziel hat [23].

Die HIV-Meldedaten aus Deutschland ähneln denen anderer EU/EWR-Mitgliedsstaaten in Bezug auf die Verteilung der Herkunftsregionen. Von 2007-2011 wurden EU/EWR-weit 125.225 HIV-Neudiagnosen gemeldet, von diesen waren 60 \% Nicht-Migranten. Die größte Gruppe von Personen mit einem anderen Herkunftsland als das berichtende Land des
Aufenthalts waren auch hier Personen aus Subsahara-Afrika (21\%), gefolgt von Personen aus Lateinamerika (4\%) [5].

\section{Syphilis}

Migration ist für die Epidemiologie von Syphilis in Deutschland bei einem Anteil von durchschnittlich $13 \%$ aller Meldungen mit Angabe einer nicht-deutschen Herkunft von eher geringerer Bedeutung. Jedoch wurden folgende herkunftsspezifische Unterschiede deutlich: So war bei Nicht-Deutschen der Frauenanteil deutlich höher, wie auch der Anteil heterosexuell übertragener Infektionen.

Die erhobenen Daten für Syphilis zeigen, dass Maßnahmen zur Prävention, Diagnostik und Therapie von Syphilis in Deutschland durchaus migrationssensibel gestaltet sein sollten, nicht zuletzt, um eventuell aufgrund kulturell bedingter Zugangsbarrieren noch nicht diagnostizierte Infektionen adäquat behandeln zu können. Umfängliche migrationssensible Kampagnen, wie dies für HIV angebracht ist, erscheinen im Fall der Syphilis nicht notwendig. Allerdings weist der höhere Anteil von im Ausland durch Inanspruchnahme von Prostitution erworbenen Infektionen auf einen entsprechenden Präventionsbedarf in Risikogruppen unabhängig von einem Migrationshintergrund hin.

Die Syphilis-Meldedaten aus Deutschland ähneln denen zahlreicher anderer europäischer Länder in Bezug auf den eher niedrigen, über die Jahre konstanten Anteil von Migranten und bei diesen auf die größere Bedeutung heterosexuell erworbener Infektionen sowie den geringeren Anteil von MSM [5].

\section{Einschätzung der Meldedaten}

Die hier beschriebene epidemiologische Lage von TB, HIV und Syphilis ergibt sich aus einer komplexen Interaktion verschiedener Faktoren. Dazu gehören nicht nur die weltweite Verteilung des Vorkommens der jeweiligen Erreger, die Inzidenz der Erkrankungen bzw. Infektionen im Herkunftsland und die Migrationsintensität aus diesem Land nach Deutschland, sondern auch Gesundheitsrisiken während des Migrationsprozesses und im Aufnahmeland, Risikoverhalten, Zugangsmöglichkeiten zu Gesundheits- versorgung in Deutschland, Diagnoseverhalten von Ärzten und Laboren sowie sozioökonomische Faktoren. Viele dieser Faktoren werden im Surveillance-System nicht erfasst.

Bei der Erhebung von Indikatoren des Migrationsstatus in der infektionsepidemiologischen Surveillance ist entsprechend ein verantwortungsvoller Umgang in der Erfassung, Auswertung und Berichterstattung der Information wesentlich und unter ethischen Gesichtspunkten gefordert, unter anderem um einer medialen Verselbständigung nicht korrekter Bilder von „Migrationskrankheiten“ und Stigmatisierungen vorzubeugen [24].

\section{Neue Surveillance-Ansätze, Studien und weiterer Forschungsbedarf}

Gesetzliche Meldedaten können zwar erste, vorsichtig zu interpretierende Hinweise auf besonders betroffene Gruppen geben, für eine tiefergehende Analyse der zugrundeliegenden Ursache und mögliche Lösungsansätze sind jedoch ergänzende Studien erforderlich.

Für ein genaueres Verständnis des Einflusses von Migration auf die Epidemiologie von Infektionskrankheiten erscheint eine Adjustierung nach Alter, Geschlecht und (momentan nicht in den Meldedaten erhobenem) sozialem Status sinnvoll. Außerdem würden Angaben zum Zeitpunkt der Einreise bzw. die Tatsache, ob regelmäßige Reisen in die Herkunftsregion erfolgen, bessere Rückschlüsse darauf erlauben, welche Rolle die eigene Migrationserfahrung spielt und ob der Infektionsort in Deutschland oder im Herkunfts- bzw. Geburtsland zu vermuten ist. Dies würde auch eine bessere Einschätzung erlauben, inwieweit $\mathrm{z}$. B. bei TB die aktuelle Resistenzsituation im Geburtsland prädiktiv ist für Resistenzmuster bei im Ausland geborenen TB-Patienten in Deutschland. Auch die Bedeutung der sich verändernden Migrationsbewegungen für die Krankheitslast von Infektionskrankheiten ist für Deutschland noch nicht eingehend untersucht.

Ergänzende Studien zu Wissen, Einstellungen und Verhalten werden benötigt, besonders für schwer erreichbare Gruppen. Nur so können detaillierte Informationen zu Präventionsbedarfen, Ri- 


\begin{tabular}{|c|c|c|c|c|c|c|c|}
\hline Studie & Population & Erreger & Gebiet & Studiendesign & $\begin{array}{l}\text { Biol. } \\
\text { Proben }\end{array}$ & Merkmale zu Migration & Laufzeit \\
\hline EMIS & MSM & $\begin{array}{l}\text { HBV, } \\
\text { HCV, } \\
\text { HIV, STI }\end{array}$ & Europa & $\begin{array}{l}\text { KABa-Studie, Inter- } \\
\text { net-Survey }\end{array}$ & Nein & $\begin{array}{l}\text { Geburtsland, Aufenthaltsdauer } \\
\text { in Deutschland }\end{array}$ & $\begin{array}{l}1 / 2009 \\
12 / 2010\end{array}$ \\
\hline $\begin{array}{l}\text { BORDER } \\
\text { NETwork II }\end{array}$ & $\begin{array}{l}\text { Klienten von HIV/STI- } \\
\text { Beratungsstellen in den } \\
\text { beteiligten Ländern }\end{array}$ & HIV, STI & $\begin{array}{l}\text { Bulgarien, Öster- } \\
\text { reich, Rumänien, } \\
\text { Slowakei }\end{array}$ & $\begin{array}{l}\text { Sentinel-Surveillan- } \\
\text { ce KAB-Studie }\end{array}$ & $\mathrm{Ja}$ & $\begin{array}{l}\text { Staatsangehörigkeit, Geburts- } \\
\text { land, Dauer Aufenthalt im } \\
\text { Land der Erhebung, Kenntnisse } \\
\text { der Sprache des Landes der } \\
\text { Erhebung }\end{array}$ & $\begin{array}{l}1 / 2010- \\
12 / 2012\end{array}$ \\
\hline $\begin{array}{l}\text { HIV 1-Sero- } \\
\text { kon-verter- } \\
\text { studie }\end{array}$ & $\begin{array}{l}\text { HIV-Patienten mit } \\
\text { bekanntem Serokonver- } \\
\text { sionszeitpunkt }\end{array}$ & HIV & Deutschland & Kohortenstudie & $\mathrm{Ja}$ & Herkunftsland & $\begin{array}{l}\text { 6/1997-lau- } \\
\text { fend }\end{array}$ \\
\hline $\begin{array}{l}\text { KABP- } \\
\text { Surv-STI }\end{array}$ & $\begin{array}{l}\text { Sexarbeiterinnen, die Ge- } \\
\text { sundheitsamt aufsuchen }\end{array}$ & HIV, STI & Deutschland & $\begin{array}{l}\text { KAB-Studie, Quer- } \\
\text { schnittsstudie }\end{array}$ & $\mathrm{Ja}$ & Geburtsland, Sprachkenntnisse & $\begin{array}{l}1 / 2010- \\
12 / 2011\end{array}$ \\
\hline STI-HIT & $\begin{array}{l}\text { Klienten der HIV/STI-Bera- } \\
\text { tungsstellen in NRW }\end{array}$ & STI & $\begin{array}{l}\text { Nordrhein- } \\
\text { Westfalen }\end{array}$ & $\begin{array}{l}\text { Prävalenzstudie, } \\
\text { Querschnittsstudie }\end{array}$ & $\mathrm{Ja}$ & Geburtsland & $\begin{array}{l}1 / 2012- \\
12 / 2014\end{array}$ \\
\hline $\begin{array}{l}\text { STI-Outre- } \\
\text { ach }\end{array}$ & $\begin{array}{l}\text { Sexarbeiter/innen, die } \\
\text { durch aufsuchende Arbeit } \\
\text { erreicht wurden }\end{array}$ & STI & $\begin{array}{l}\text { Berlin, Hamburg } \\
\text { Nordrhein- } \\
\text { Westfalen }\end{array}$ & $\begin{array}{l}\text { Prävalenzstudie, } \\
\text { Querschnittsstudie }\end{array}$ & Ja & $\begin{array}{l}\text { Geburtsland, Deutschkennt- } \\
\text { nisse }\end{array}$ & $\begin{array}{l}1 / 2012- \\
12 / 2014\end{array}$ \\
\hline Sialon II & $\begin{array}{l}\text { MSM, die an Szeneorten } \\
\text { in Hamburg erreicht } \\
\text { wurden }\end{array}$ & HIV & Hamburg & $\begin{array}{l}\text { Prävalenzstudie, } \\
\text { Querschnittsstudie, } \\
\text { KAB-Studie, Time } \\
\text { Location Sampling } \\
\text { (TLS) }\end{array}$ & Ja & $\begin{array}{l}\text { Geburtsland, Land des aktuel- } \\
\text { len Aufenthalts }\end{array}$ & $\begin{array}{l}12 / 2011- \\
6 / 2015\end{array}$ \\
\hline $\begin{array}{l}\text { DRUCK- } \\
\text { Studie }\end{array}$ & i. v. Drogengebraucher & $\begin{array}{l}\text { HIV, } \\
\text { HBV, } \\
\text { HCV, } \\
\text { HTLV-1, } \\
\text { HTLV-2 }\end{array}$ & $\begin{array}{l}\text { Berlin, Es- } \\
\text { sen, Leipzig, } \\
\text { Frankfurt/M., } \\
\text { Köln, Hannover, } \\
\text { München, Ham- } \\
\text { burg }\end{array}$ & $\begin{array}{l}\text { KAB-Studie, Prä- } \\
\text { valenzstudie, } \\
\text { Querschnittsstudie, } \\
\text { Respondent Driven } \\
\text { Sampling (RDS) }\end{array}$ & $\mathrm{Ja}$ & $\begin{array}{l}\text { Geburtsland der Teilnehmer } \\
\text { und deren Eltern }\end{array}$ & $\begin{array}{l}4 / 2012- \\
3 / 2015\end{array}$ \\
\hline MiSSA & $\begin{array}{l}\text { Migranten aus Subsahara- } \\
\text { Afrika }\end{array}$ & $\begin{array}{l}\text { HBV, } \\
\text { HCV, } \\
\text { HIV, STI }\end{array}$ & $\begin{array}{l}\text { Hamburg } \\
\text { (Pilotstudie), } \\
\text { München, } \\
\text { Rhein-Ruhr- } \\
\text { Region, Berlin, } \\
\text { Frankfurt/M., } \\
\text { Hannover }\end{array}$ & $\begin{array}{l}\text { KAB-Studie, Quer- } \\
\text { schnittsstudie, } \\
\text { partizipativer For- } \\
\text { schungsverlauf }\end{array}$ & Nein & $\begin{array}{l}\text { Geburtsland, Geburtsland der } \\
\text { Eltern, Aufenthaltsdauer in } \\
\text { Deutschland, Sprachkennt- } \\
\text { nisse }\end{array}$ & $\begin{array}{l}\text { 5/2013- } \\
5 / 2014 \text { (Pi- } \\
\text { lotstudie) } \\
9 / 14-12 / 16\end{array}$ \\
\hline $\begin{array}{l}\text { SMA-Stu- } \\
\text { die }\end{array}$ & MSM & HIV, STI & Deutschland & $\begin{array}{l}\text { KAB-Studie, Quer- } \\
\text { schnittsstudie, } \\
\text { Internet-Survey }\end{array}$ & $J a^{b}$ & $\begin{array}{l}\text { Land des aktuellen Aufent- } \\
\text { halts, Geburtsland der Teilneh- } \\
\text { mer, der Mutter, des Vaters }\end{array}$ & $\begin{array}{l}\text { 6/2013- } \\
9 / 2014\end{array}$ \\
\hline \multicolumn{8}{|c|}{$\begin{array}{l}\text { HBV Hepatitis B, HCV Hepatitis C, HTLV Humanes T-lymphotropes Virus, } i \text {. v. intravenös, MSM Männer, die Sex mit Männern haben, STI sexuell übertragbare Infektionen } \\
\text { (sexually transmittable infetions) } \\
\text { aKAB: Studien, die Daten zu Knowledge (Wissen), Attitudes (Einstellungen) und Behaviour (Verhalten) erheben }\end{array}$} \\
\hline
\end{tabular}

siko- und Schutzverhalten generiert werden.

Solche Studien werden am RKI insbesondere zu sexuell oder durch Blut übertragbare Infektionen bereits durchgeführt (- Tab. 4). Art und Ausmaß der Erhebung von migrationsspezifischen Merkmalen unterscheiden sich zwischen den Studien, was oft den jeweils spezifischen Zugangsund Erhebungsbedingungen der jeweiligen Studienpopulationen geschuldet ist. Im Anschluss an diese Studien gilt es weiter zu untersuchen, wie die dargestell- ten Gruppen am effektivsten mit Präventionsstrategien erreicht werden, wo Barrieren bestehen und wie diese abgebaut werden können.

\section{Limitationen}

Die Auswertung der Meldedaten erfolgte rein deskriptiv. Die quantitativen Ergebnisse basieren auf den an das RKI gemeldeten bzw. übermittelten Daten. Diese können einer Unter- und Fehlerfassung unterliegen. Ebenso beinhalten die
Meldedaten keine weiteren Faktoren, die mit dem Migrationsstatus verknüpft sein könnten, wie sozialer Status. Festgestellte Unterschiede sind somit nicht notwendigerweise kausal auf den Migrationshintergrund zurückzuführen, und möglicherweise sind nicht alle tatsächlich bestehenden Unterschiede sichtbar.

\section{Fazit}

Innerhalb der infektionsepidemiologischen Surveillance in Deutschland sowie 
auf europäischer Ebene werden unterschiedliche Indikatoren zur Erfassung eines Migrationshintergrundes verwendet. Eine Vereinheitlichung der erhobenen Indikatoren und Anpassung an den empfohlenen Indikator "Geburtsland" würde sowohl europäische Vergleiche als auch Vergleiche zwischen verschiedenen Infektionskrankheiten bzw. Erregern ermöglichen. Die zusätzliche Erhebung der Staatsangehörigkeit würde in Deutschland außerdem die Berechnung von Inzidenzen für verschiedene Bevölkerungsgruppen ermöglichen - wie bei TB bereits durchgeführt.

Die aktuell in der Surveillance genutzten Indikatoren zur Erfassung des Migrationsstatus ermöglichen zum einen das Erkennen von Entwicklungen, z. B. in der anteiligen Zusammensetzung von Geburtsländern der TB-Patienten. Zum anderen erlauben sie die Identifikation von Gruppen mit besonderem Risikoprofil, wie TB-Patienten aus NUS hinsichtlich multiresistenter Formen der TB. Sie geben weiterhin Hinweise auf Präventionsund Therapiebedarfe, wie beispielsweise für Migranten aus Subsahara-Afrika, die sich in Deutschland mit HIV infiziert haben.

Entlang dieser Hinweise aus der infektionsepidemiologischen Surveillance ist begleitende und ergänzende Forschung dringend notwendig, um die komplexen Zusammenhänge von Migration und Infektionskrankheiten richtig zu interpretieren und sich an der Zielpopulation orientierende Public-Health-Maßnahmen gestalten zu können.

\section{Korrespondenzadresse}

\section{A. Kuehne}

Abteilung für Infektionsepidemiologie

Robert Koch-Institut, Seestraße 10

13353 Berlin

KuehneAn@rki.de

\section{Santos-Hövener}

Abteilung für Infektionsepidemiologie

Robert Koch-Institut, Seestraße 10

13353 Berlin

Santos-HoevenerC@rki.de
Danksagung. Wir danken den Kolleginnen und Kollegen der Fachgebiete 31, 32, 34 und 36 des Robert Koch-Instituts für ihre Unterstützung, die Verarbeitung und die Bereitstellung der Meldedaten, sowie allen Personen und Institutionen, die zur infek tionsepidemiologischen Surveillance in Deutschland beitragen. Ebenso danken wir den Fachgebietsleitern Andreas Gilsdorf, Viviane Bremer und Walter Haas für ihre Unterstützung bei der Erstellung dieses Artikels. Schließlich möchten wir uns bei der abteilungsübergreifenden Arbeitsgruppe (Abteilung 2 und 3) zu Migration bedanken, durch die die Idee zu diesem Artikel entstanden ist.

\section{Einhaltung ethischer Richtlinien}

Interessenkonflikt. Anna Kuehne, Lena Fiebig, Klaus Jansen, Carmen Koschollek, Claudia SantosHövener geben an, dass kein Interessenskonflikt vorliegt.

Dieser Beitrag beinhaltet keine Studien an Menschen oder Tieren.

\section{Literatur}

1. Statistische Ämter des Bundes und der Länder (2013) Bevölkerung nach Migrationsstatus regional. Ergebnisse des Mikrozensus 2011. Statistische Ämter des Bundes und der Länder, Wiesbaden

2. Schenk L, Bau A-M, Borde T, Butler J, Lampert T et al (2006) Mindestindikatorensatz zur Erfassung des Migrationsstatus. Bundesgesundheitsbl Gesundheitsforsch Gesundheitsschutz 49:853-860

3. Schenk L, Ellert U, Neuhauser H (2007) Kinder und Jugendliche mit Migrationshintergrund in Deutschland. Bundesgesundheitsbl Gesundheitsforsch Gesundheitsschutz 50:590-599

4. Akmatov MK, Mikolajczyk RT, Krumkamp R, Wörmann T, Chu JJ et al (2012) Availability of indicators of migration in the surveillance of HIV, tuberculosis and hepatitis B in the European Union - a short note. J Public Health 20:483-486

5. ECDC (2014) Technical report: assessing the burden of key infectious diseases affecting migrant populations in the EU/EEA - executive summary. ECDC, Stockholm

6. Ott JJ, Stevens GA, Groeger J, Wiersma ST (2012) Global epidemiology of hepatitis $B$ virus infection: New estimates of age-specific HBsAg seroprevalence and endemicity. Vaccine 30:2212-2219

7. WHO (2014) Global tuberculosis report. WHO, Genf

8. WHO, UNAIDS, UNICEF (2011) Global HIV/AIDS response: epidemic update and health sector progress towards universal access: progress report 2011. WHO, Genf

9. ECDC (2012) Responses to HIV and migration in western industrialised countries. ECDC, Stockholm

10. Robert Koch-Institut (2008) Migration und Gesundheit. Robert Koch-Institut, Berlin

11. Robert Koch-Institut (2014) Bericht zur Epidemiologie der Tuberkulose in Deutschland für 2013. Robert Koch-Institut, Berlin

12. Robert Koch-Institut (2012) HIV bei Migranten in Deutschland. Epidemiol Bull 3:19-21

13. Robert Koch-Institut (2014) HIV-Infektionen und AIDS-Erkrankungen in Deutschland - Bericht zur Entwicklung im Jahr 2013 aus dem Robert KochInstitut. Epidemiol Bull 213-230
14. ECDC (2011) Technical report: improving HIV data comparability in migrant populations and ethnic minorities in EU/EEA/EFTA countries: findings from a literature review and expert panel. ECDC, Stockholm

15. Statistisches Bundesamt (2014) Bevölkerung und Erwerbstätigkeit, Ausländische Bevölkerung, Ergebnisse des Ausländerzentralregisters. Statistische Bundesamt, Wiesbaden

16. Schenk L, Neuhauser H (2005) Methodische Standards für eine migrationssensible Forschung in der Epidemiologie. Bundesgesundheitsbl Gesundheitsforsch Gesundheitsschutz 48:279-286

17. Marx F, Fiebig L, Hauer B, Brodhun B, Glaser-Paschke $G$ et al (2014) Higher rate of tuberculosis in second generation migrants compared to native residents in a metropolitan setting in Western Europe. PLoS One (angenommen zur Publikation)

18. Karo B, Haas W, Kollan C, Gunsenheimer-Bartmeyer B, Hamouda O et al (2014) Tuberculosis among people living with HIV/AIDS in the German ClinSurv HIV cohort: long-term incidence and risk factors. BMC Infect Dis 14:148

19. Fiebig L, Kollan C, Hauer B, Gunsenheimer-Bartmeyer B, an der Heiden M et al (2012) HIV-prevalence in tuberculosis patients in Germany, 20022009: an estimation based on HIV and tuberculosis surveillance data. PLoS One 7:e49111

20. Angell SY, Behrens RH (2005) Risk assessment and disease prevention in travelers visiting friends and relatives. Infect Dis Clin North Am 19:49-65

21. Koschollek C, Santos-Hövener C (2012) Mapping afrikanischer Communities in Deutschland. Eine Analyse von Daten des Statistischen Bundesamtes. Robert Koch-Institut, Berlin. http://www.rki.de/DE/ Content/InfAZ/H/HIVAIDS/Studien/MiSSA/Pilotstudie_Mapping.pdf?_blob=publicationFile

22. Rice BD, Elford J, Yin Z, Delpech VC (2012) A new method to assign country of HIV infection among heterosexuals born abroad and diagnosed with HIV. AIDS 26:1961-1966

23. Santos-Hövener C, Marcus U, Koschollek C, Oudini $\mathrm{H}$, Wiebe M et al (2014) Konzeption einer Studie zu sexueller Gesundheit bei in Deutschland lebenden Afrikanern. Prävent Gesundheitsförd 1-8

24. Kehr J (2010) Geographien der Gefahr. Wie Tuberkulose in Deutschland zu einer Migrantenkrankheit wird. In: Dilger H, Hadolt B (Hrsg) Medizin im Kontext Krankheit und Gesundheit in einer vernetzten Welt. Peter Lang GmbH; Internationaler Verlag der Wissenschaften, Frankfurt a. M. http:// www.peterlang.com/index.cfm?event $=$ cmp.ccc. seitenstruktur.detailseiten\&seitentyp=produkt $\&$ $\mathrm{pk}=51618$ 